\title{
Herpes Zoster Oticus With Delayed Facial Palsy and Multiple Cranial Nerve Involvement
}

\author{
Kyung Min Kim, Kwiju Yu, Eun-Ju Jeon, and Hyun Jin Lee 10 \\ Department of Otolaryngology-Head and Neck Surgery, College of Medicine, The Catholic University of Korea, Seoul, Korea
}

지연성 안면 마비 및 다발성 뇌신경 마비를 동반한 이성대상포진

김경민 · 유귀주 · 전은주 · 이현진

가톨릭대학교 의과대학 이비인후과학교실

\author{
Received March 8, 2021 \\ Revised May 20, 2021 \\ Accepted June 3, 2021 \\ Address for correspondence \\ Hyun Jin Lee, MD \\ Department of Otorhinolaryngology- \\ Head and Neck Surgery, \\ Incheon St. Mary's Hospital, \\ College of Medicine, \\ The Catholic University of Korea, \\ 56 Dongsu-ro, Bupyung-gu, \\ Incheon 21431, Korea \\ Tel +82-32-280-5151 \\ Fax $+82-32-280-5821$ \\ E-mail idgenesis@naver.com
}

Herpes zoster oticus with multiple cranial nerve $(\mathrm{CN})$ involvement and delayed facial paralysis is a rare occurrence, and there is limited information regarding the disease. We herein report two cases of RHS with delayed facial palsy involving multiple lower CNs. The degree of facial paralysis was not severe as grade III or less, and recovery was observed after treatment. To our knowledge, these are the first cases in the literature to report RHS with delayed facial paralysis and its favorable recovery. It will be a good example for expanding the possibility of treatment in RHS with delayed facial paralysis and multiple $\mathrm{CN}$ involvement.

Korean J Otorhinolaryngol-Head Neck Surg 2021;64(11):839-43

Keywords Facial palsy; Ramsay Hunt syndrome; Varicella zoster virus.

\section{Introduction}

Ramsay Hunt syndrome (RHS), caused by infection with the varicella zoster virus (VZV), is characterized by ipsilateral otalgia, vesicular skin rash around the auricle and auditory canal, and peripheral facial palsy. ${ }^{1)}$ It is caused by reactivation of the VZV in the geniculate ganglion and the adjacent motor branches of the facial nerve. VZV reactivation within the cranial nerve $(\mathrm{CN})$ or dorsal root ganglia is associated with a wide range of neurological presentations and complications. It can invade the adjacent $\mathrm{CN}$ and cause further neurologic disturbance. ${ }^{2)}$ RHS with multiple lower $\mathrm{CN}$ involvement occurs rarely in only $1.8 \%$ of all RHS patients. ${ }^{3)}$ It is known that various symptoms of paralysis occur simultaneously with the

This is an Open Access article distributed under the terms of the Creative Commons Attribution Non-Commercial License (https://creativecommons.org/licenses/by-nc/4.0) which permits unrestricted non-commercial use, distribution, and reproduction in any medium, provided the original work is properly cited. onset of RHS. ${ }^{4)}$ In contrast, RHS with delayed facial palsy and multiple $\mathrm{CN}$ involvement is a sufficiently rare occurrence. Recently, one case of delayed CN IX and X palsy that occurred 16 days after CN V, VII, and VIII palsy was reported, but there has been no report of RHS with delayed onset facial palsy. We herein report two cases of RHS with delayed facial palsy involving multiple lower CNs.

\section{Case}

\section{Case 1}

A 24-year-old male presented with a 3-day history of vertigo, along with a 2-day history of left-side otalgia, sore throat, and hoarseness. A few vesicles were observed on the left external auditory canal and tympanic membrane. Several vesicles were also observed on the left soft palate and the uvula was deviated to the right during phonation. The gag reflex on 
the left side was also decreased. Telelaryngoscopic examination revealed erythema and white exudate covering the mucosa over the left arytenoid, accompanied by vocal cord palsy. Right-beating spontaneous nystagmus was grade III, as well as $39 \%$ caloric weakness on the left side was revealed on videonystagmography. There was also mild sensory neural hearing loss in the left ear. On serologic test, the immunoglobulin (Ig)M antibody against VZV was positive, whereas the IgG antibody was negative. MRI with gadolinium enhancement of the brain revealed no abnormal lesions. Under the impression of herpes zoster oticus with multiple $\mathrm{CN}$ involvement (VIII, IX, X), oral famciclovir and prednisolone therapy were applied. Oral steroid (Solondo ${ }^{\circledR}$, prednisolone; Yuhan Corporation, Seoul, Korea) was used $60 \mathrm{mg} /$ day for 5 days, then tapered during next 5 days. Oral famciclovir (Famvics ${ }^{\circledR}$, famciclovir; Yuhan Corporation) was used $750 \mathrm{mg} /$ day for 7 days. For dizziness control, Alevert $^{\circledR}$ (dimenhyrinate $40 \mathrm{mg}$ with cinnarizine 20 mg; Unipharm Korea, Seoul, Korea) was used twice a day. After 2 weeks, delayed left-side facial paralysis (HouseBrackmann grade II) occurred (Fig. 1A). We applied additional steroid pulse therapy for delayed facial paralysis. Steroids were used at the same dose as the initial treatment. Dizziness, ear fullness, and facial paralysis in the patient were improved after additional steroid pulse therapy (Fig. 1B). Pure tone audiometry showed $10 \mathrm{~dB}$ of hearing gain after two months, which is in normal range. However, vocal cord palsy still persisted as intermediate palsy state after 6 months (Fig. 2A).

\section{Case 2}

A 52-year-old female was admitted to our clinic with sore throat, globus sensation, and dysphagia for 2 days. She had diabetes mellitus type 2 and chronic hepatitis B infection. On the telelaryngoscopic examination, several vesicles were revealed on the right side of the pharynx, accompanied by right vocal cord palsy (Fig. 2B). A physical examination revealed vesicular eruption on the right-sided auricle. The patient did
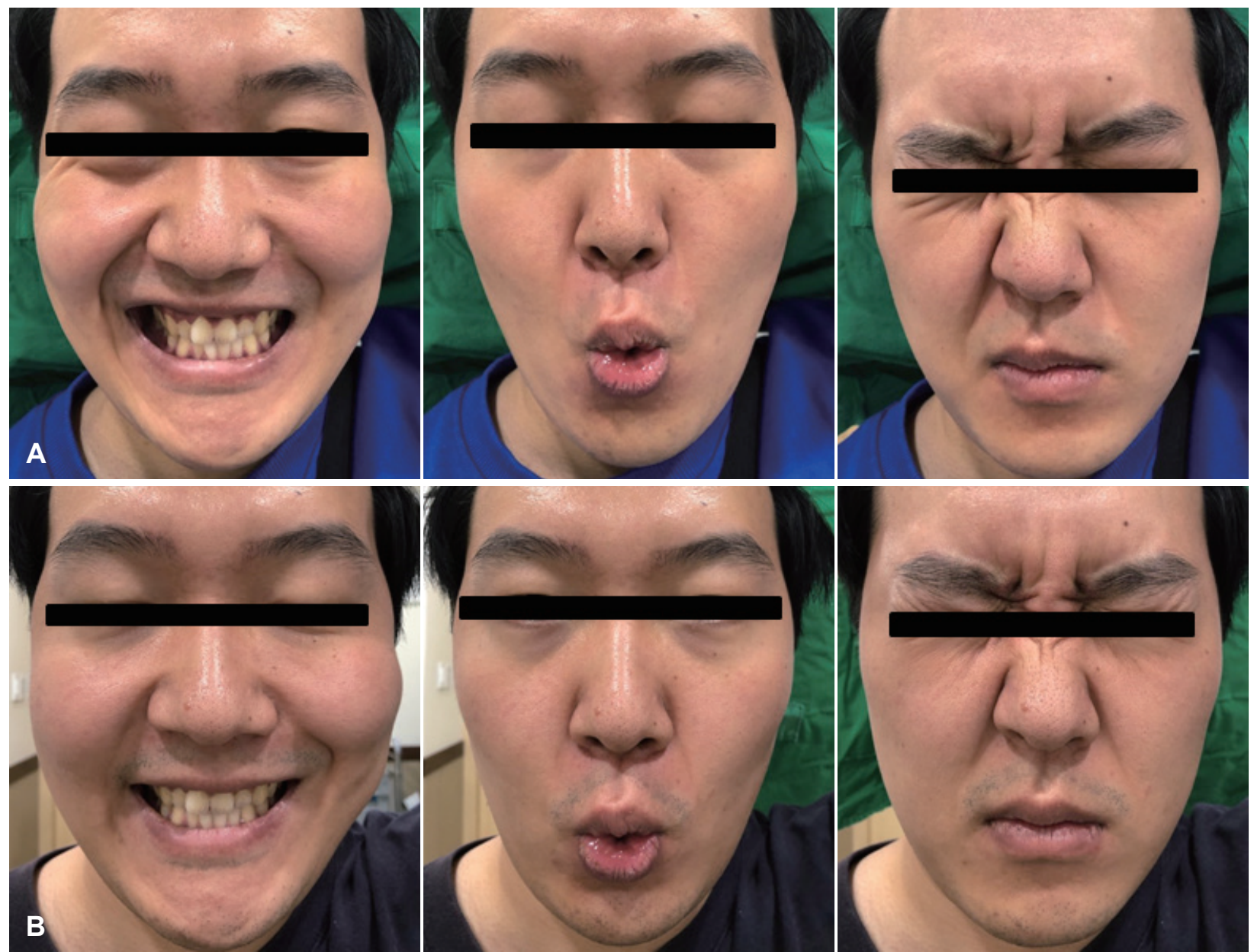

Fig. 1. Facial paralysis in case 1. A: Delayed left-sided facial paralysis (House-Brackmann grade II) occurred after 2 weeks. B: Facial paralysis was improved after additional steroid pulse therapy. 

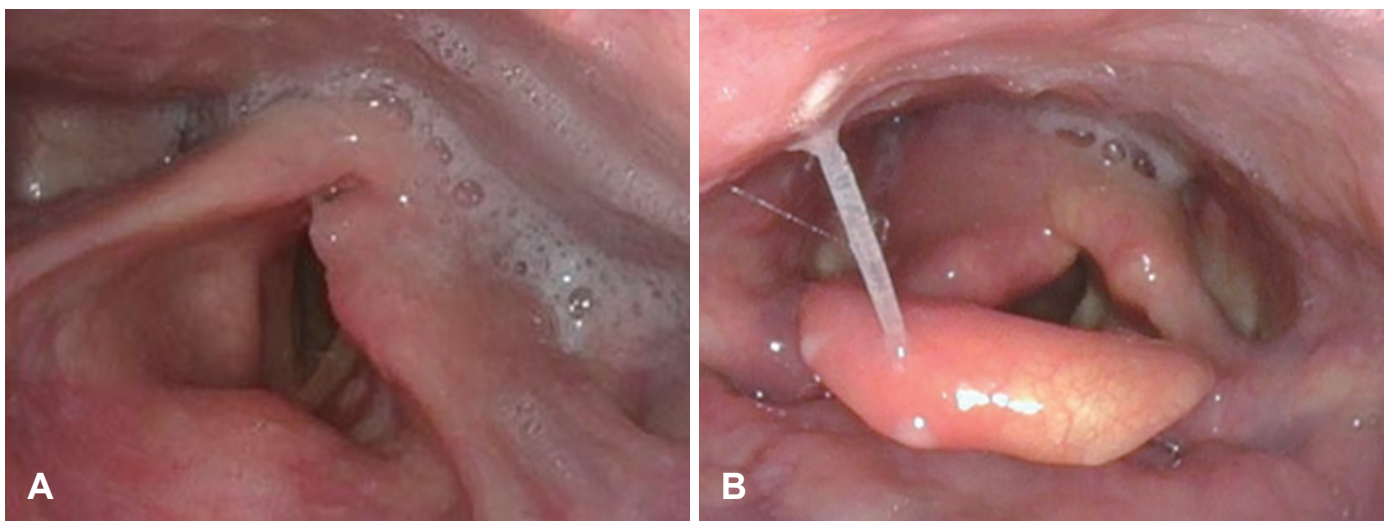

Fig. 2. Initial telelaryngoscopic images. A: Case 1 showed left vocal cord palsy. B: Case 2 showed right vocal cord palsy.

not have any other symptoms, such as otalgia, facial weakness, hearing impairment, or dizziness. No definite abnormal findings were found on neck and chest CT scan and brain-temporal MRI. On serologic test, $\operatorname{IgM}$ and $\operatorname{IgG}$ antibodies against varicella-zoster were negative. However, a serum polymerase chain reaction test for VZV was positive. The patient was given intravenous (IV) acyclovir and high dose prednisolone therapy. Oral steroid (Solondo ${ }^{\circledR}$, prednisolone) was used $60 \mathrm{mg} /$ day for 5 days, then tapered during next 5 days. IV antiviral agent (Zoylex $^{\circledR}$, acyclovir; Korea United Pharm, Seoul, Korea) was used $750 \mathrm{mg} /$ day for 5 days and changed to oral agent (Famvics $^{\circledR}$, famciclovir) $750 \mathrm{mg} /$ day for next 2 days. At 5 days after therapy, vocal cord palsy and pharyngeal vesicles were improved and the patient was discharged without any complication. However, three weeks from symptom onset, the patient began to complain of an additional symptom of right-sided facial palsy of House-Brackmann grade III. There was no vesicle or skin lesion on the right ear. Therefore, additional prednisolone with acyclovir therapy was given for one week. Steroids were used at the same dose as the initial treatment, and IV antiviral agent $\left(\right.$ Zoylex $^{\circledR}$, acyclovir) was used $750 \mathrm{mg} /$ day for 5 days. Then, two weeks after facial paralysis, sudden tinnitus in the right side and vertigo occurred. On neurologic examination, spontaneous nystagmus to the left side of grade II was revealed and videonystagmography showed $69 \%$ of caloric weakness on the right side. Hearing impairment was not observed on pure tone audiometry. Since, there were no evidence to suggest the central origin lesion with physical examination and videonystagmography, serial MRI was not taken. Under the impression of delayed RHS with multiple neuropathies, we started one more cycle of acyclovir and prednisolone therapy. After 6 months, right-sided facial palsy and vocal cord palsy recovered completely and the patient did not have any dizziness, tinnitus, dysphagia, or skin lesion.

\section{Discussion}

RHS with multiple CN involvement and delayed facial paralysis is a rare occurrence, and there is limited information regarding the disease. Both patients fully recovered from delayed facial paralysis, which was the characteristic in both cases. Multiple CN palsy was improved in the two cases except for right vocal cord palsy in case 1 . In the previous case reports, polyneuritis may occur ascending, descending on the progress, or not described. ${ }^{6}$ From this point of view, the course of symptoms may vary from person to person; therefore, the symptom manifestation might be different in the two cases. However, delayed facial palsy was not severe in both cases; it was below House-Brackmann grade III, and they recovered.

RHS is an infectious disease caused by the VZV. ${ }^{1)}$ The reason for the slowly developed facial paralysis was probably the result of reactivation of the VZV that remained dormant in the nerve root ganglion. Analysis of the VZV DNA in the dorsal root ganglia from a person with zoster showed that viral DNA was present in both the neurons and satellite cells of ganglia innervating the sites of reactivation. ${ }^{7)}$ Close anatomic relationship between CN can cause the symptom of polyneuropathy. Trigeminal and vestibulocochlear nerves and spinal ganglia $\mathrm{C} 2-\mathrm{C} 4$ are known to be the commonly affected nerves. ${ }^{8)} \mathrm{Se}-$ vere inflammation in one ganglion can spread to another adjacent ganglion. It can also cause infarction by invading microvascular structures that have a common distribution in other brain nerves. VZV can directly invade the brainstem parenchyma by way of nerve axons or via synapses. ${ }^{9)}$ Recently, a hypothesis that, VZV causes synaptic conduction along the reflex pathways of the brainstem, causing multiple neuropathy, has also been raised. ${ }^{10)}$

The etiology of facial paralysis in RHS may be caused by VZV infection. The facial nerve is composed of two sensory 
branches. They are responsible for the general sense of the pinna and the taste of the tongue. The VZV may invade the sensory branches of the facial nerve and penetrate to the geniculate ganglion. When the virus is reactivated, the inflammation of adjacent motor branches of the facial nerve leads to facial paralysis. This phenomenon explains the mechanism of RHS (facial nerve palsy with zoster oticus). ${ }^{11)}$

On serologic test, case 1 showed a positive result for $\operatorname{IgM}$ and a negative result for $\mathrm{IgG}$, while both $\mathrm{IgM}$ and $\mathrm{IgG}$ were negative in case 2 . The positive rate of serum VZV IgM in patients with RHS was reported to be significantly higher (30.8\%) than that in patients with Bell's palsy $(9.8 \%){ }^{12)}$ Serologic screening for IgG against VZV will aid in identifying nonimmune individuals. The presence of IgM against VZV is suggestive of an acute or recent infection. However, results should be correlated with the clinical presentation because the patient's symptoms are the most important criteria in RHS diagnosis.

RHS with multiple $\mathrm{CN}$ palsy has rarely been reported. ${ }^{13-15)}$ Kim, et al. ${ }^{3)}$ reported 11 patients with 10 years of RHS with polyneuritis, and the frequency of $\mathrm{CN}$ involvement was VII, VIII, IX, X, and V in that order. Shim, et al. ${ }^{13)}$ reported 11 cases for 15 years, and the following CN VII, VIII nerves, the frequency of other $\mathrm{CN}$ was $\mathrm{CN} \mathrm{X}, \mathrm{IX}$, and V in that order. In a paper analyzing RHS with polyneuritis combined with vocal cord palsy $(\mathrm{CN} \mathrm{X})$ reported in the literature for 50 years; of the 14 patients, the incidence was CN VII, VIII, IX, V, and XII. ${ }^{4)}$ There are few prospective controlled studies for RHS with multiple $\mathrm{CN}$ involvement in the medical literature. The actual management starts with a combination of an antiviral agent and steroids. We used an antiviral agent and steroids as the initial treatment. In the event of delayed facial paralysis, an additional antiviral and steroid treatment was applied. After the second treatment, delayed facial paralysis recovered, which means that delayed infection is considered to have a lower level of inflammation. Therefore, immediate antiviral agent or steroid treatment is required when recurrent symptoms appear.

RHS is known to have a poorer prognosis than Bell's palsy. Peitersen reported the recovery rate in 1701 cases of Bell's palsy and 116 cases of herpes zoster. In the Bell's palsy group, recovery was found in $83 \%$ of patients, but the recovery rate was only $46 \%$ in the RHS group and the remaining patients showed moderate to high sequelae. The prognosis of multiple cranial neuritis is still controversial. Kim, et al. ${ }^{3)}$ reported that facial palsy was improved satisfactorily, and the remaining symptoms except for CN VIII recovered well. Shim, et al. ${ }^{13)}$ reported that only 6 of the 11 patients had improved symptoms, and the recovery rate of facial palsy (54.5\%) was worse than that in the simple RHS group (82.9\%). Rasmussen reported that recovery of vocal cord paralysis was found in only 4 of 14 patients (28.6\%), and facial paralysis was the same. ${ }^{4)}$ The above studies showed a difference in the recovery rate when facial paralysis occurred in the early stages. However, in our cases, the degree of facial paralysis was not severe as grade III or less, and recovery was observed after treatment.

To our knowledge, these are the interesting cases in the literature to report RHS with delayed facial paralysis and its favorable recovery. It will be a good example for expanding the possibility of treatment in RHS with multiple CN palsy.

In conclusion, delayed facial paralysis can develop in RHS with multiple cranial polyneuritis after several weeks from the onset. Therefore, physicians should keep in mind the possibility of delayed facial paralysis and the need for proper treatment.

\section{Acknowledgments}

This work was supported by a National Research Foundation (NRF) of Korea grant funded by the Korean government (MSIT) (2020R1C1C1005965).

\section{Author Contribution}

Conceptualization: Kwiju Yu, Eun-Ju Jeon, Hyun Jin Lee. Data curation: Kyung Min Kim, Hyun Jin Lee. Formal analysis: Eun-Ju Jeon, Hyun Jin Lee. Funding acquisition: Hyun Jin Lee. Investigation: Hyun Jin Lee. Project administration: Hyun Jin Lee. Resources: Kwiju Yu, Hyun Jin Lee. Supervision: Eun-Ju Jeon, Hyun Jin Lee. Validation: Hyun Jin Lee. Visualization: Hyun Jin Lee. Writing — original draft: Kyung Min Kim, Hyun Jin Lee. Writing-review \& editing: Kyung Min Kim, Kwiju Yu, Hyun Jin Lee.

\section{ORCID}

Hyun Jin Lee

https://orcid.org/0000-0003-3826-8830

\section{REFERENCES}

1) Hunt JR. On herpetic inflammations of the geniculate ganglion. A new syndrome and its complications. J Nerv Ment Dis 1907;34(2): 73-96.

2) Sweeney CJ, Gilden DH. Ramsay Hunt syndrome. J Neurol Neurosurg Psychiatry 2001;71(2):149-54.

3) Kim YH, Chang MY, Jung HH, Park YS, Lee SH, Lee JH, et al. Prognosis of Ramsay Hunt syndrome presenting as cranial polyneuropathy. Laryngoscope 2010;120(11):2270-6.

4) Rasmussen ER, Mey K. Vocal cord paralysis associated with Ramsay Hunt syndrome: Looking back 50 years. BMJ Case Rep 2014;2014:bcr2013201038.

5) Kim JM, Lee Z, Han S, Park D. Treatment of Ramsay-Hunt's syndrome with multiple cranial nerve involvement and severe dysphagia: A case report. Medicine (Baltimore) 2018;97(17):e0591.

6) Hwang W, Jeong DE. Zoster laryngitis with multiple cranial nerve palsy progressed as ascending involvement. J Neurocrit Care 2018; 11(1):43-6.

7) Lungu O, Annunziato PW, Gershon A, Staugaitis SM, Josefson D, LaRussa $P$, et al. Reactivated and latent varicella-zoster virus in 
human dorsal root ganglia. Proc Natl Acad Sci U S A 1995;92(24): 10980-4.

8) Wagner G, Klinge H, Sachse MM. Ramsay Hunt syndrome. J Dtsch Dermatol Ges 2012;10(4):238-44.

9) Hu S, Walker M, Czartoski T, Cheng A, Forghani B, Gilden DH, et al. Acyclovir responsive brain stem disease after the Ramsay Hunt syndrome. J Neurol Sci 2004;217(1):111-3.

10) Alicandri-Ciufelli M, Aggazzotti-Cavazza E, Genovese E, Monzani D, Presutti L. Herpes zoster oticus: A clinical model for a transynaptic, reflex pathways, viral transmission hypotheses. Neurosci Res 2012;74(1):7-9.

11) Grose $C$, Bonthius D, Afifi AK. Chickenpox and the geniculate ganglion: Facial nerve palsy, Ramsay Hunt syndrome and acyclovir treatment. Pediatr Infect Dis J 2002;21(7):615-7.

12) Shin HW, Choi YS, Moon SJ, Song JJ, Kim CH, Lee JH, et al. Viral serologic test in sudden sensorineural hearing loss and acute peripheral facial paralysis: Is it always necessary? Korean J Otorhinolaryngol-Head Neck Surg 2008;51(12):1088-92.

13) Shim HJ, Jung H, Park DC, Lee JH, Yeo SG. Ramsay Hunt syndrome with multicranial nerve involvement. Acta Otolaryngol 2011;131(2):210-5.

14) Shinha $T$, Krishna P. Ramsay Hunt syndrome and zoster laryngitis with multiple cranial nerve involvement. IDCases 2015;2(2):47-8.

15) Lee DH, Yoon TM, Lee JK, Joo YE, Lim SC. Herpes zoster laryngitis accompanied by Ramsay Hunt syndrome. J Craniofac Surg 2013;24(5):e496-8. 